\section{A black swan?}

\author{
Mike Mew \\ mikemew@gmail.com
}

Send your comments to the

Editor-in-Chief

British Dental Journal

64 Wimpole Street,

London

W1G 8YS

Email bdj@bda.org
In the last one hundred years there has been in-depth and extensive research into the practice of orthodontics. Huge advances have been made, however there still remains scope to further explore key aspects of orthodontic theory. Arguably, several pertinent facts have escaped investigation:

- There is still no known cause (aetiology) in most cases of malocclusion

- There is no cure and permanent retention is now standard

- There is little evidence that orthodontic treatment provides a long term benefit.

Without a universal theory, orthodontists in the same country prescribe widely varying treatment plans and different countries are philosophically poles apart. Understandably, this is of concern but there are limitations on experimentation on children and in the absence of a comprehensive philosophy, orthodontics has leaned towards empiricisms. Given this position it is our duty as professionals to ensure a fair and impartial hearing to any proposed theory, if only to exclude them. Karl Popper suggested that it is not possible to prove anything but only to see which theory fits the facts best.

The proposed theory of orthotropics suggests that environmental factors cause malocclusion and the genes decide its pattern. Primarily, our mandibular motor tone has reduced as we have moved from a high fibre, low nutritional diet to a high nutrient, low fibre diet, at the same time that we require fewer calories and have a general reduction in body motor tone. Secondarily, allergies causing transient nasal obstructions lead to a long term postural lowering of the mandible. Children with an open mouth posture and lowered mandibular motor tone tend to have longer faces. As the body of the mandible, the length of which is under tight genetic control, angles downwards then the horizontal or tooth bearing section shortens. The expected result is crowding at the extremities, the wisdom teeth and the incisors (the lowers being affected most as they are trapped under a falling maxilla). Unfortunately, fixed appliances increase the vertical growth which is the very problem. The strength of co-habitational effects should not be underestimated and families sharing the same diet, environment and genes are likely to be affected in the same way.

\section{MALOCCLUSION IS A SIGN OF CIVILIZATION}

In the fields of both anthropology and archaeology malocclusion is considered a sign of civilization. It is not present in any of our pre-civilised ancestors who had well aligned teeth (regardless of attrition) from birth until death. The origin of malocclusions does not follow a genetic pattern but appears wherever and whenever cooking and tool making appear, and progresses from mild imbrications to skeletal manifestations as civilization develops. There is also no evidence that we should not accommodate 32 well aligned teeth today or that there has been any genetic change.

This is a great over simplification. For a theory to be tested it must be heard in its entirety. The entire topic of orthodontics could not be subjected to an investigation at one lecture and neither could an alternative philosophy. It is difficult clinically to compare a system that primarily aims to align teeth with one that aims to improve faces, and relies entirely on the cooperation of 8-year-old children. Orthotropics believes that teeth have the ability to align themselves if the face grows well, as they do in the other 4,629 different species of mammals, and trying to align them artificially has a poor long term prognosis. Orthotropics produces the best facial changes in the world; it has also successfully treated 20-30 children who were told that they would need surgery. Clinicians in specialist practice do not produce large random controlled trials, nor should they. It should be for universities funded by the public to investigate for the public good, if only to prove orthotropics wrong. This is what MScs and PhDs are for.

There is no better method to align teeth than contemporary orthodontics but should this be our only goal? It is not for us to decide but the parents of the children that we treat and without this information they are being denied informed consent. To general dentists who are convinced that malocclusion is genetic I implore you to let the evidence speak for itself; this is why evidence-based medicine was developed, to allow science to challenge preconceptions.

This challenges the best paid and most politically well organised section of our profession, and I ask for constructive feedback only. Neither side has the evidence to validate themselves, but one theory must fit the evidence better than the other. There is however, adequate evidence to debate the smaller subject of the aetiology of malocclusion. I challenge the orthodontic profession to a debate to test the hypothesis that 'Malocclusion is caused by the environment and modified by the genes'; if this is upheld then an independent investigation of the theory of orthotropics should ensue.

DOI: 10.1038/sj.bdj.2009.325 